\title{
A comparison between acute pressure block of the sciatic nerve and acupressure: methodology, analgesia, and mechanism involved
}

This article was published in the following Dove Press journal:

Journal of Pain Research

25 July 2013

Number of times this article has been viewed

\section{Danping Luo ${ }^{1,2}$ \\ Xiaolin Wang' \\ Jiman $\mathrm{He}^{1,3}$}

'Pain Medicine Program, Nanfang Hospital, Southern Medical University,

Guangzhou, People's Republic of China; ${ }^{2}$ The First Affiliated Hospital of Guangdong College of Pharmacy, Guangzhou, People's Republic of China; ${ }^{3}$ Department of Medicine, Brown University, Providence, RI, USA

Correspondence: Jiman $\mathrm{He}$

Pain Medicine Program, Nanfang Hospital, Southern Medical University, People's

Republic of China

Tel +86206278622I

Fax +862087280770

Email jiman_he@brown.edu

\begin{abstract}
Acupressure is an alternative medicine methodology that originated in ancient China. Treatment effects are achieved by stimulating acupuncture points using acute pressure. Acute pressure block of the sciatic nerve is a newly reported analgesic method based on a current neuroscience concept: stimulation of the peripheral nerves increases the pain threshold. Both methods use pressure as an intervention method. Herein, we compare the methodology and mechanism of these two methods, which exhibit several similarities and differences. Acupressure entails variation in the duration of manipulation, and the analgesic effect achieved can be shortor long-term. The acute effect attained with acupressure presents a scope that is very different from that of the chronic effect attained after long-term treatment. This acute effect appears to have some similarities to that achieved with acute pressure block of the sciatic nerve, both in methodology and mechanism. More evidence is needed to determine whether there is a relationship between the two methods.
\end{abstract}

Keywords: acupressure, acute pressure block, sciatic nerve, pain

\section{Background}

Acupressure is an alternative medicine methodology that originated in ancient China and is a popular therapy worldwide. Acupressure involves application of acute pressure on the acupuncture points to achieve treatment effects that are similar to those of the acupuncture method. ${ }^{1-3}$ According to traditional Chinese medicine (TCM), stimulating these points can correct imbalance in the flow of qi through channels known as meridians, rebalancing yin and yang and subsequently treating diseases. ${ }^{4}$ Instead of using a thin needle to stimulate the specific points, acupressure uses the fingers, elbow, fist, etc to stimulate the acupuncture points. Acupressure displays therapeutic effects for several types of diseases. There are several types of pressure points; pressure to each is applied differently, and each creates a different effect accordingly. ${ }^{5}$

Acute pressure block of the sciatic nerve is a newly reported analgesic method, ${ }^{6-10}$ based on a current neuroscience concept: stimulation of the peripheral nerves increases the pain threshold. The method works well to alleviate various types of clinical pain but not experimental cold pressor pain or intense pain. ${ }^{6,7,9}$ Some physicians, patients, and scientists have interpreted this method as another approach for delivering the previously described analgesic method, acupressure. Herein, we compare the methodology and mechanism of these two methods. An understanding of these aspects is important for their application in clinical practice and improvement of their efficacy.

The theoretical target objects of the two methods are different. Acupressure uses pressure to stimulate specific acupuncture points for therapeutic purposes. ${ }^{4}$ In contrast, 
acute pressure block of the sciatic nerve method uses pressure to stimulate the sciatic nerve. ${ }^{6-9}$ The stimulation site is an area rather than a point, and stimulation of a larger area provides better pain relief. Many acupuncture points are located over nerves (Figure 1). Thus, pressure over a larger area may cause stimulation of several points. Future studies are required to examine the rationale behind them.

These methods entail different degrees of pressure application. The optimal method for applying acupressure focuses on a point, using, for example, the thumbs, fingers, elbows, or suitable devices; however, the optimal method for acute pressure block of the sciatic nerve is to apply pressure to a larger area, using, for example, the palms of the hands, the flat surfaces of the fists, or suitable devices.

The amount of pressure required is usually approximately $10 \mathrm{~kg}$ to $20 \mathrm{~kg}$ per hand area for a man of average size. ${ }^{6-9}$ Therefore, although the thumbs, fingers, or elbows are ideal for stimulating the acupuncture points in the acupressure method, they are not ideal for acute pressure block of the sciatic nerve, because the area required for this method is much larger. These reasons may indicate that the mechanisms involved in the two methods are different.

Theoretically, each acupuncture point usually is effective for one or a few types of diseases, ${ }^{5}$ whereas acute pressure block of the sciatic nerve has been shown to work for

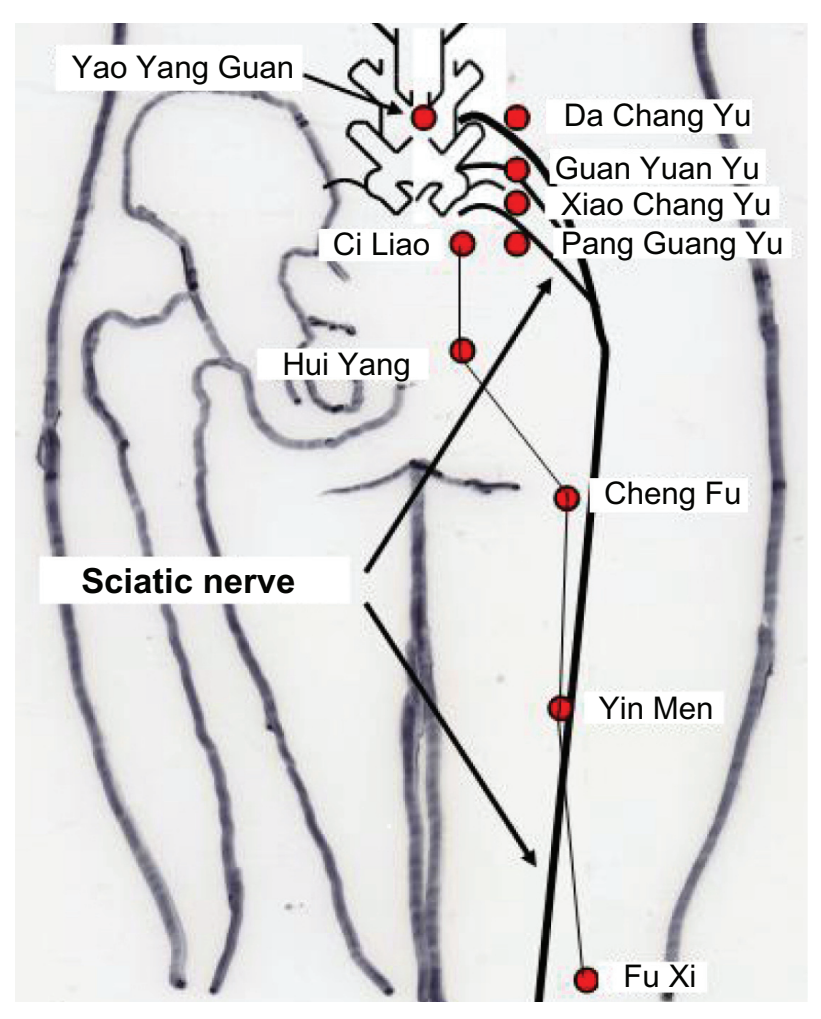

Figure I The sciatic nerve and some acupuncture points. more general areas of the body. In TCM, a disease is generally perceived as disharmony (or an imbalance) in the functions or interactions of the meridians, such as yin, yang, xue, and zang-fu. ${ }^{11}$ Therefore, the appropriate therapy is recommended on the basis of which "pattern of disharmony" can be identified. ${ }^{12,13}$ There are 360 acupuncture points, ${ }^{14}$ each with its own therapeutic significance. The selection of which point to target is a key step in acupressure practice, and selection of the correct acupuncture point is essential to achieve good treatment outcomes. ${ }^{5}$ Various principles are proposed for selecting these points. ${ }^{15}$ For example, a common method for choosing acupuncture points is to select nearby points, which means points are selected in a local area that is adjacent to the disease-affected area.

Although the only target of acute pressure block of the sciatic nerve method is the sciatic nerve, this treatment provides relief from various pains in different areas of the body. According to reports, the acute pressure block method is most effective for dental pain, which originates far from the sciatic nerve, compared with pain resulting from other diseases. ${ }^{6,8}$ This observation shows that the working mechanisms may be different for the two methods.

Acupuncture is usually considered to not have a physically verifiable anatomic or histological basis, whereas the acute pressure block method has an associated anatomic structure, the sciatic nerve. Acupuncture points are located along meridians that connect acupuncture points across the anatomy and affect a specific organ or other part of the body. TCM recognizes 360 points and twelve "regular" and eight "extraordinary" meridians. ${ }^{16}$ In addition, a number of less customary channels branching off from the regular meridians have been recognized. ${ }^{17}$ A 1997 National Institutes of Health consensus ${ }^{4}$ stated that "Despite considerable efforts to understand the anatomy and physiology of the 'acupuncture points,' the definition and characterization of these points remains controversial". Scientific research has not found any histological or physiological correlates for qi, meridians, or acupuncture points. ${ }^{18-20}$ However, the method of acute pressure block of the sciatic nerve has an associated anatomic structure, the sciatic nerve. The sciatic nerve is large and the longest single nerve in humans.

The mechanisms underlying the analgesic effect of the acupressure and the acute pressure block of the sciatic nerve methods may be different. Traditional Chinese acupuncture is a philosophy that focuses more on prevention than treatment of illnesses. In the general theory of TCM, bodily functions are regulated by an energy called qi, which flows through the body. Acupuncture points are mainly located at specific 
locations along the meridians. The meridians are believed to connect the various parts of the entire body and to associate with the yin, yang, and qi. Yin and yang are opposite motions that are interdependent of each other. Disruptions to the flow of qi along the meridians will cause diseases, ${ }^{4,21}$ and stimulating these points can regulate the meridian system, restore the balance of qi, yin, and yang, and subsequently help the body to recover from diseases., ${ }^{4,21}$

Current scientific studies on the mechanism of action of these acupuncture points have suggested that the insertion of needles involves recruitment of the body's own pain reduction system, possibly accompanied by an increased release of endorphins, serotonin, norepinephrine, or $\gamma$-Aminobutyric acid. ${ }^{4,22}$ However, endogenous opioids and other molecular secretions need time to reach their effective level. Although acupressure on some points can generate a faster analgesic effect (within a few minutes), ${ }^{23-25}$ the mechanism for this faster analgesic effect remains unknown.

The mechanism for acute pressure block of the sciatic nerve is based in general on current progress in neurobiology: Stimulation of peripheral nerves increases the pain threshold. ${ }^{26-28}$ Chemical, ${ }^{29}$ mechanical, ${ }^{3}$ or electrical $^{31}$ block of the sciatic nerve has reportedly generated analgesic effects. According to the gate control theory of pain, ${ }^{31}$ stimulation of large-diameter afferent fibers inhibits second-order neurons in the dorsal horn and prevents the further transmission of impulses carried by small-diameter fibers; the resulting analgesic effect is considered rapid and short-lived and is thought to involve spinal dorsal horn wide dynamic range neurons. ${ }^{31-33}$ Wide dynamic range neurons are the first synaptic relay point for afferent pathways and play an important role in modifying the transmission of noxious input. ${ }^{34,35}$ A recent study showed that acute pressure on the rat sciatic nerve caused immediate inhibition of wide dynamic range neuron responses. ${ }^{10}$ Thus, the gate control theory of pain may explain the immediate analgesic effect of the acute pressure block of the sciatic nerve method.

Sensory transduction in nerves is accomplished by ion channel proteins, which are gated pores that allow the exchange of ions across the cell membrane. Acid-sensitive ion channel proteins, which were shown to be expressed in the neurons of the mammalian central and peripheral nervous systems, are proposed to constitute mechanoreceptors and to play an important role in responses to mechanical stimuli. ${ }^{36-39}$ To date, four acid-sensitive ion channel proteins have been found to be expressed in the sciatic nerve. ${ }^{40}$ Thus, the molecular mechanism of pain inhibition by acute pressure block of the sciatic nerve may involve pressure activation of acid-sensitive ion channel proteins.

The time required to generate the analgesic effect differs between the two methods. The time required to achieve a treatment effect by activation of the acupuncture points varies greatly depending on the nature of the disease being treated. A common course of treatment may initially involve dozens of treatments spaced at approximately weekly intervals that are later extended to monthly intervals. ${ }^{41-42}$ Acupressure stimulation of some acupuncture points can also exert acute effects that last several minutes to several hours after a single treatment. ${ }^{43}$ For example, acupressure on the acupuncture point yao yang guan can provide relief of acute sciatica pain ${ }^{13}$ and requires long-term treatment for an effect. ${ }^{44,45}$ However, the time required for acute pressure block of the sciatic nerve to generate an analgesic effect is only a few minutes. ${ }^{6-10}$ The marked difference in the time required to generate treatment effects may be attributable to differences in the mechanisms between the two methods.

More evidence is needed to clarify the effectiveness of the two methods in other diseases. According to TCM, energy (qi) flow through the body is essential for health, and disruptions of this flow are believed to be responsible for various diseases. Stimulating acupuncture points removes blocks in qi and helps it flow back into balance. Acupressure is considered a potentially useful strategy for the management of multiple symptoms. Therapeutic effects on various diseases have been studied, including different pain pathologies, nausea, osteoarthritis, vomiting, rheumatoid arthritis, allergic rhinitis, and insomnia. The results of these studies are controversial. Although consistent data on the positive effects of acupressure therapy have been reported for some conditions, such as low back pain, nausea, and vomiting, ${ }^{46-47}$ the evidence for its positive effects on other pathological conditions is not conclusive.

A report from the American Academy of Pain Medicine's 24th Annual Meeting ${ }^{48}$ shows that acupressure can work on non-painful discomfort, such as fullness, bloating, nausea, and vomiting; the method has a smaller effect on non-painful discomfort than it does on pain..$^{48}$ However, more studies are needed to confirm these results.

\section{Is there a relationship between acupressure and acute pressure block of sciatic nerve method?}

Both methods involve application of acute pressure to achieve treatment effects. Both methods alleviate pain and discomfort from a number of diseases. Are these observations attribut- 
able to mere coincidence, or does a relationship exist between the two methods?

Yao yang guan (or waist yang guan), is beneficial for acute sciatica pain and low back pain. This acupuncture point is located below the spinous process of the fourth lumbar vertebra, where branches of the sciatic nerve extend from the spinal cord (Figure 1). As seen in a few studies, effective pressure on any accessible area along the sciatic nerve will provide rapid pain relief, but the effectiveness is reduced if the pressure is applied distant from the sciatic nerve. ${ }^{6,7,9}$ Is the relief from sciatica pain attributable to stimulation of the acupuncture point or to stimulation of the sciatic nerve?

Acupuncture points shown on the TCM map ${ }^{49-51}$ are found in areas close to the nerve. Figure 1 displays the sciatic nerve through the low back and leg, as well as the distribution of some acupuncture points on the lower back and leg. Of note, the locations of the sciatic nerve path and the acupuncture points are close to each other. This raises the question of whether acupressure exerts its treatment effect via stimulation of adjacent nerves. Studies have shown that direct stimulation of peripheral nerve sensory fibers increases the pain threshold in a manner similar to that caused by the standard acupuncture technique. ${ }^{52,53}$ Although current scientific research may explain some aspects of TCM, it is far from understanding of the old theory, which was passed down from generation to generation.

\section{Conclusion}

The present report compares the major similarities and differences between acupressure and acute pressure block of the sciatic nerve. Treatment effects for acupressure are achieved with great variation in duration. Accordingly, the underlying mechanism appears to vary greatly. The acute effect attained with acupressure presents a scope that is very different from that of the chronic effect attained after longterm treatment. This effect appears to have more similarities to that achieved with acute pressure block of the sciatic nerve, both in methodology and mechanism. Many aspects of the two methods are unknown, and more evidence is therefore required to determine whether a relationship exists between the two. Further studies aimed at clarifying this relationship are important for improvements in the efficacy of these two methods.

\section{Acknowledgments}

No external funding was obtained for this project. This work was solely supported by department sources.

\section{Author contributions}

Jiman He conceived the study, wrote the manuscript, and had final approval of the version to be published. Danping Luo and Xiaolin Wang wrote the manuscript, revised the content, and had final approval of the version to be published.

\section{Disclosure}

The authors report no conflicts of interest in this work.

\section{References}

1. Nepp J, Wedrich A. Conjuctivitis sicca: a comparison of traditional Chinese and Western medical symptoms. Acupunct Med. 1994;12(2): $88-92$.

2. Bertrand SW. Inroads to integrative health care: registered nurses' personal use of traditional Chinese medicine affects professional identity and nursing practice. $J$ Evid Based Complementary Altern Med. 2010;15(1):14-30.

3. Dundee JW. Studies with acupuncture/acupressure as an antiemetic. Acupunct Med. 1988;5:22-24.

4. Acupuncture. National Institute of Health Consensus Development Conference Statement. November 3-5, 1997;15(5):1-34. Available at http://consensus.nih.gov/1997/1997acupuncture107html.htm. Accessed July $3,2013$.

5. Li Z. The point, Yao Yang Guan, prevents acute sciatica pain. In: Small Acupuncture Points, Big Health. Chinese Science Culture Publishing House; 2009

6. He J, Jiang X, Zhao B, et al. Acute pressure block of the sciatic nerve relieves clinical pain but not cold pressor pain. Clin J Pain. 2010;26(4): 332-338.

7. He J, Wu B, Jiang X, Zhang F, Zhao T, Zhang W. A new analgesic method, two-minute sciatic nerve press, for immediate pain relief: a randomized trial. BMC Anesthesiol. 2008;8:1.

8. He J, Chen Q. General responses to questions regarding acute sciatic nerve pressure for pain relief. Pain Med. 2010;11(7):1139-1140.

9. He J, Wu B, Zhang W, Ten G. Immediate and short-term pain relief by acute sciatic nerve press: a randomized controlled trial. $B M C$ Anesthesiol. 2007;7:4

10. Wang W, Tan W, Luo D, et al. Acute pressure on the sciatic nerve results in immediate inhibition of the wide dynamic range neuronal response. BMC Neurosci. 2012;13:147.

11. Wiseman N, translator-editor; Ellis A, translator. Fundamentals of Chinese Medicine. Taos, NM: Paradigm Publications; Saint Paul, MN, USA. 1996.

12. Ergil MC, Ergil KV. Pocket Atlas of Chinese Medicine. Stuttgart: Thieme; 2009.

13. Flaws B, Finney D. A Handbook of TCM Patterns and Their Treatments, 6th ed. Boulder, CO: Blue Poppy Press; Boulder, CO, USA. 2007.

14. Lu G-D, Needham J. Celestial Lancets: A History and Rationale of Acupuncture and Moxa. New York: Routledge; 2002.

15. McDonald J. Missing the point: a discussion of strategies for acupuncture of point selection. Pacific J Oriental Med. 1999;15:26-38.

16. Lin Y. Map of Jing luo. Fujian, China: Fujian Science and Technology Publishing House; Fujian, People's Republic of China. 1985.

17. Aung SKH, Chen WPD. Clinical Introduction to Medical Acupuncture. New York: Thieme Medical Publishers; 2007.

18. Bauer M. The final days of traditional beliefs? Part one. Chin Med Times. 2006;1:31.

19. Ahn AC, Colbert AP, Anderson BD, et al. Electrical properties of acupuncture points and meridians: a systematic review. Bioelectromagnetics. 2008;29(4):245-256.

20. Mann F. Reinventing Acupuncture: A New Concept of Ancient Medicine. London: Butterworth Heinemann; 1996. 
21. Singh S, Ernst E. The truth about acupuncture. In: Trick or Treatment: The Undeniable Facts About Alternative Medicine. New York: Norton and Co; 2008:39-90.

22. Sun Y, Gan TJ, Dubose JW, Habib AS. Acupuncture and related techniques for postoperative pain: a systematic review of randomized controlled trials. Br J Anaesth. 2008;101(2):151-160.

23. Kober A, Scheck T, Greher M, et al. Prehospital analgesia with acupressure in victims of minor trauma: a prospective, randomized, double-blinded trial. Anesth Analg. 2002;95(3):723-727.

24. Hsieh LL, Kuo CH, Yen MF, Chen THH. A randomized controlled clinical trial for low back pain treated by acupressure and physical therapy. Prev Med. 2004;39(1):168-176.

25. Hsieh LL, Kuo CH, Lee LH, Yen AM, Chien KL, Chen TH. Treatment of low back pain by acupressure and physical therapy: randomised controlled trial. BMJ. 2006;332(7543):696-700.

26. Chung JM, Lee KH, Hori Y, Endo K, Willis WD. Factors influencing peripheral nerve stimulation produced inhibition of primate spinothalamic tract cells. Pain. 1984;19(3):277-293.

27. Hanai F. Effect of electrical stimulation of peripheral nerves on neuropathic pain. Spine (Phila Pa 1976). 2000;25(15):1886-1892.

28. JørumE. The analgesic effect of peripheral nerve stimulation in various tests of nociception in rats. Acta Physiol Scand.1988;133(2):131-138.

29. Sotgiu ML, Biella G, Riva L. A study of early ongoing activity in dorsal horn units following sciatic nerve constriction. Neuroreport. 1994;5(18):2609-2612.

30. Yao T, Andersson S, Thoren P. Long-lasting cardiovascular depressor response following sciatic stimulation in spontaneously hypertensive rats: evidence for the involvement of central endorphin and serotonin systems. Brain Res. 1982;244(2):295-303.

31. Melzack R, Wall PD. Pain mechanisms: a new theory. Science. 1965;150(3699):971-979.

32. Wall PD. Dorsal horn electrophysiology. In: Handbook of Sensory Physiology: Somatosensory System. Iggo A, editor. Berlin: SpringerVerlag; 1973;2:253-270.

33. Cohen ML, Arroyo JF, Champion GD, Browne CD. In search of the pathogenesis of refractory cervicobrachial pain syndrome: a deconstruction of the RSI phenomenon. Med J Aust. 1992;156(6): 432-436.

34. Coghill RC, Mayer DJ, Price DD. Wide dynamic range but not nociceptive-specific neurons encode multidimensional features of prolonged repetitive heat pain. J Neurophysiol. 1993;69(3):703-716.

35. Schaible HG. Spinal mechanisms contributing to joint pain. Novartis Found Symp. 2004;260:4-22.

36. Geffeney SL, Goodman MB. How we feel: ion channel partnerships that detect mechanical inputs and give rise to touch and pain perception. Neuron. 2012;74(4):609-619.

37. Höger U, French AS. Extracellular acid increases the open probability of transduction channels in spider mechanoreceptors. Eur J Neurosci. 2002;16(12):2311-2316.
38. Kang S, Jang JH, Price MP, et al. Simultaneous disruption of mouse ASIC1a, ASIC2 and ASIC3 genes enhances cutaneous mechanosensitivity. PLoS One. 2012;7:e35225.

39. Price MP, Thompson RJ, Eshcol JO, Wemmie JA, Benson CJ. Stomatin modulates gating of acid-sensing ion channels. J Biol Chem. 2004;279(51):53886-53891.

40. Alvarez de la Rosa D, Zhang P, Shao D, White F, Canessa CM. Functional implications of the localization and activity of acid-sensitive channels in rat peripheral nervous system. Proc Natl Acad Sci USA. 2002;99(4):326-331.

41. Itoh K, Kitakoji H. Effects of acupuncture to treat fibromyalgia: a preliminary randomized controlled trial. Chin Med. 2010;5:11.

42. Hsieh LL, Liou HH, Lee LH, Chen TH, Yen AM. Effect of acupressure and trigger points in treating headache: a randomized controlled trial. Am J Chin Med. 2010;38(1):1-14.

43. Mirbaqher-Ajorpaz N, Adib-Hajbaghery M, Mosaebi F. The effects of acupressure on primary dysmenorrhea: a randomized controlled trial. Complement Ther Clin Pract. 2011;17(1):33-36.

44. Wollaston DE, Xu X, Tokumaru O, Chen JD, McNearney TA. Patients with systemic sclerosis have unique and persistent alterations in gastric myoelectrical activity with acupressure to Neiguan point PC6. J Rheumatol. 2005;32(3):494-501.

45. Pirrie W. Acupressure. Br Med J. 1867;2(348):171-177.

46. Griffiths JD, Gyte GM, Paranjothy S, Brown HC, Broughton HK, Thomas J. Interventions for preventing nausea and vomiting in women undergoing regional anaesthesia for caesarean section. Cochrane Database Syst Rev. 2012;9:CD007579.

47. Allais G, Rolando S, Castagnoli Gabellari I, et al. Acupressure in the control of migraine-associated nausea. Neurol Sci. 2012;33 Suppl 1: S207-S210.

48. He J. Immediate relief of non-painful discomfort with an acute sciatic nerve press. Pain Med. 2008;9:88-141. Available from http://aapm.confex.com/aapm/2008am/techprogram/P2949.HTM. Accessed July 17, 2013.

49. Xu G. Applied Acupuncture Points. Shenyang. Science Tech Publishing; 2009.

50. Gang W, Li S, Huang Y, et al. Standard acupuncture appoint WHO. People Publishing; Beijing, People's Republic of China. 2010:89-111.

51. Hesketh T, Zhu WX. Health in China: traditional Chinese medicine: one country, two systems. BMJ. 1997;315(7100):115-117.

52. Lim TW, Loh T, Kranz H, Scott D. Acupuncture: effect on normal subjects. Med J Aust. 1977;1(13):440-442.

53. Chen L, Tang J, White PF, et al. The effect of location of transcutaneous electrical nerve stimulation on postoperative opioid analgesic requirement: acupoint versus nonacupoint stimulation. Anesth Analg. 1998;87(5):1129-1134
Journal of Pain Research

\section{Publish your work in this journal}

The Journal of Pain Research is an international, peer-reviewed, open access, online journal that welcomes laboratory and clinical findings in the fields of pain research and the prevention and management of pain. Original research, reviews, symposium reports, hypothesis formation and commentaries are all considered for publication.

\section{Dovepress}

The manuscript management system is completely online and includes a very quick and fair peer-review system, which is all easy to use. Visit http://www.dovepress.com/testimonials.php to read real quotes from published authors. 